\title{
Première partie \\ Outils de diagnostic et émergence des maladies infectieuses aquacoles
}

\begin{abstract}
L'apparition d'une maladie infectieuse en aquaculture est associée à des conditions zootechniques et environnementales données. De ce fait, le recueil des commémoratifs est absolument indispensable pour orienter le diagnostic et choisir les outils diagnostiques adaptés. Par ailleurs, pour répondre de manière efficace au problème de maladies émergentes infectieuses en aquaculture, il est nécessaire de disposer de réseaux de surveillance possédant un maillage assez fin et d'outils de diagnostic efficaces.
\end{abstract}

Le premier article de synthèse (Michel et al) présente les intérêts et les limites des principales techniques diagnostiques en santé des espèces aquacoles. Dans le domaine du diagnostic des maladies rencontrées en aquaculture, un triple constat s'impose : le manque de techniques de diagnostic sensibles et spécifiques validées (développement d'outils dans différents laboratoires souvent sans concertation), une absence de laboratoire référent en termes de diagnostic des maladies des poissons et un besoin de reconnaissance des techniques sérologiques en pathologie des poissons dans un cadre réglementaire.

Le deuxième article de synthèse (Pépin et al) fait le point sur l'émergence de maladies chez des organismes non aquacoles. Les maladies infectieuses émergentes ou réémergentes sont principalement caractérisées par une augmentation brutale de leur incidence et par une incertitude sur l'ampleur du phénomène.

Deux articles plus spécifiques donnent des exemples d'émergence de maladies infectieuses aquacoles chez les poissons et les mollusques (Saulnier et al, Douet). De nombreux cas de maladies infectieuses émergentes ont en effet été rapportés ces dernières décennies en aquaculture : infection herpétique de la carpe Koï, infections impliquant des bactéries du genre Vibrio chez les crevettes en Nouvelle-Calédonie, bonamiose de l'huître plate en Europe, infections à nodavirus. Ces cas montrent que les causes et les facteurs favorisant l'émergence de maladies infectieuses peuvent être multiples : apparition d'un nouvel agent infectieux, évolution d'un agent existant, introduction d'un agent pathogène exotique, notion de vecteurs et de transferts, évolution des techniques de diagnostic et effet de facteurs environnementaux. Conserver des échantillons de manière adéquate afin de pouvoir les analyser de nouveau lorsqu'il y a des améliorations ou des avancées en termes de diagnostic apparaît comme une approche indispensable afin de mieux connaître les maladies infectieuses et y faire face. 
